\title{
On permutation polytopes: notions of equivalence
}

\author{
Barbara Baumeister • Matthias Grüninger
}

Received: 13 December 2013 / Accepted: 17 November 2014 / Published online: 11 December 2014

(C) Springer Science+Business Media New York 2014

\begin{abstract}
By assigning a permutation polytope to a group, we produce new interesting polytopes. For the effective use of this construction method, it is desirable to understand which groups are leading to affine equivalent polytopes. Therefore, the notion of effective equivalence has been introduced (Baumeister et al. in Adv Math 222(2):431$452,2009)$. In this note, we clarify the notion of effective equivalence and characterize it geometrically. Moreover, we present examples showing that the effective equivalent permutation groups do not correspond to affinely equivalent polytopes. We also apply the characterization to the examples. In our approach we provide a framework for the use of representation theoretic methods in the study of permutation polytopes.
\end{abstract}

Keywords Polytope - Birkhoff polytope - Permutation polytope .

Affine equivalence $\cdot$ Effective equivalence of groups

\section{Introduction}

The permutation polytopes are an interesting class of polytopes, see for instance $[1-6,8,9]$. In particular, they provide a tool to produce new interesting polytopes, which moreover can be studied with group theoretic and representation theoretic methods. Therefore, it is also important to understand which groups are leading to affine equivalent polytopes. This study has been started in [2]. Abstractly isomorphic groups do not need to have affinely equivalent permutation polytopes: For instance

\footnotetext{
B. Baumeister $(\varangle)$

University Bielefeld, Bielefeld, Germany

e-mail: b.baumeister@math.uni-bielefeld.de

M. Grüninger

University Würzburg, Würzburg, Germany

e-mail: matthias.grueninger@mathematik.uni-wuerzburg.de
} 
$\langle(12),(34)\rangle$ and $\langle(12)(34),(13)(24)\rangle$ are isomorphic groups, but the associated permutation polytopes are a quadrangle and a tetrahedron, respectively, and therefore not affinely equivalent.

On the other hand, the notion of isomorphism of permutation groups is too restrictive to describe the affine equivalent permutation polytopes. In [2], Sect. 2.1, it has been observed that there are two permutation groups which are not isomorphic as permutation groups but whose permutation polytopes are affinely equivalent: The permutation polytopes of the permutation groups

$$
\langle(1234)\rangle \leq \operatorname{Sym}(4),\langle(1234)(5)\rangle \leq \operatorname{Sym}(5) \text { and }\langle(1234)(56)\rangle \leq \operatorname{Sym}(6)
$$

are all tetrahedrons and therefore all affinely equivalent, but the underlying groups are not isomorphic as permutation groups.

The notion of isomorphism of permutation groups has been generalized to the notion of effectively equivalent permutation groups in [2]; for the definition see the next section. In fact, all the permutation groups listed in the last paragraph are effectively equivalent permutation groups.

The hope was that two permutation groups are effectively equivalent if and only if the groups are isomorphic and the corresponding permutation polytopes are affinely equivalent. Here we present an example of two permutation groups which are isomorphic as abstract groups and whose permutation polytopes are affinely equivalent, but which are not effectively equivalent thereby answering Question 2.12 of [2].

Up to now, the tools in studying permutation polytopes were mainly connected to convex geometry and group theory. In this note, we provide a representation theoretical basis for the study of these polytopes. Moreover, we use representation theory to prove a criterion which determines when two permutation groups $G_{1}$ and $G_{2}$ are effective equivalent in terms of their permutation polytopes $P\left(G_{1}\right)$ and $P\left(G_{2}\right)$ (see Theorem 4.3, Corollary 4.5 and Theorem 4.6).

The organization of the note is as follows: We start by introducing the notation and recall the relevant previous results. In the third section, we presented the examples. The representation theoretical approach is presented in the fourth section. There we also characterize the effectively equivalent groups. These results are applied to our examples in the last, the fifth, section.

\section{Notation and previous results}

The convex and the affine hull of a set $S$ in a real vector space will be denoted by $\operatorname{conv}(S)$ and by aff $(S)$, respectively.

\subsection{Permutation polytopes}

An injective homomorphism $\pi: G \rightarrow \operatorname{Sym}(n)$ is called permutation representation. The pair $(G, \pi)$ is called permutation group. In this case, we obtain a representation polytope as follows. 
The symmetric group $\operatorname{Sym}(n)$ acts on the set $\{1, \ldots, n\}$. Let $V$ be an $n$-dimensional $\mathbb{R}$-vector space with basis $\left\{e_{1}, \ldots, e_{n}\right\}$ and let $\operatorname{Sym}(n)$ act on this vector space by permuting the indices of the vectors in the basis. Then $V$ is the permutation module for $\operatorname{Sym}(n)$. This module induces a representation $R: \operatorname{Sym}(n) \rightarrow \operatorname{GL}(V)=\operatorname{GL}\left(\mathbb{R}^{n}\right)$, $g \mapsto M_{g}$, and thereby identifies the symmetric group $\operatorname{Sym}(n)$ with the set of $n \times n$ permutation matrices, i.e., the set of matrices whose entries are 0 or 1 such that in every column and every row there is a unique 1 . The polytope

$$
P(G, \pi):=\operatorname{conv}(\pi(G)) \subseteq \operatorname{Mat}_{n}(\mathbb{R}) \cong \mathbb{R}^{n \times n}
$$

is called the permutation polytope associated to $(G, \pi)$. If $\pi$ or $G$ is clear from the context then we abbreviate $P(G, \pi)$ by $P(G)$ or by $P(\pi)$.

The special case $G=\operatorname{Sym}(n)$ yields the well-known $n$th Birkhoff polytope $B_{n}:=P(\pi(\operatorname{Sym}(n)))$ (see e.g., [5]). The concept of a permutation polytope can be generalized to a representation polytope by considering any real representation of $G$ instead of a permutation representation, see [2].

\subsection{Notions of equivalence of polytopes}

For a standard reference on polytopes, we refer to [11]. If the vertices of a polytope $P \subseteq \mathbb{R}^{m}$ are a subset of a full dimensional lattice $\Lambda$ in $\mathbb{R}^{m}$, then we call $P$ a lattice polytope. In this sense, every permutation polytope is a lattice polytope, as the vertices all lie in $\operatorname{Mat}_{n}(\mathbb{Z})$.

As moreover, every vertex of a permutation polytope is a matrix whose entries are only 0 and 1 , it is also a 0/1-polytope, i.e., a polytope whose vertices are in the set $\{0,1\}^{d}$ for some $d \in \mathbb{N}$.

There are several notions of equivalence of (lattice) polytopes (see [11]):

Definition 2.1 Two polytopes $P \subset \mathbb{R}^{m}$ and $Q \subset \mathbb{R}^{n}$ are affinely equivalent if there is an affine isomorphism of the affine hulls $\phi: \operatorname{aff}(P) \rightarrow \operatorname{aff}(Q)$ that maps $P$ onto $Q$, write $P \approx_{\text {aff }} Q$. For lattice equivalence, we additionally require that $\phi$ is an isomorphism of the affine lattices (aff $P) \cap \Lambda \rightarrow($ aff $Q) \cap \Lambda^{\prime}$. Combinatorial equivalence is an equivalence of the face lattices as posets.

\subsection{Notions of equivalence of groups}

To identify permutation groups that define affinely equivalent permutation polytopes, the notion of effective equivalence has been introduced (see [2]).

We use the following non-standard notation, which does not conflict with the usual notation. For $K=\mathbb{R}$ or $\mathbb{C}$ we denote by $\operatorname{Irr}_{K}(G)$, the set of pairwise nonequivalent irreducible $K$-representations, i.e. homomorphisms $G \rightarrow \operatorname{GL}(W)$ where $W$ is a $K$-vector space which does not contain a proper $G$-invariant subspace. For instance, there is the trivial representation, $1_{G}: G \rightarrow \mathrm{GL}(K), g \mapsto 1$. Every representation $\rho: G \rightarrow \mathrm{GL}(V)$ over $K$ splits into irreducible representations. We denote the pairwise non-equivalent irreducible factors of $\rho$ by $\operatorname{Irr}_{K}(\rho) \subseteq \operatorname{Irr}_{K}(G)$. 
Definition 2.2 Two real representations $\rho_{1}$ and $\rho_{2}$ of $G$ are stably equivalent if they contain equivalent non-trivial irreducible factors. Two faithful real representations $\rho_{i}: G_{i} \rightarrow \mathrm{GL}\left(V_{i}\right)$ (for $i=1,2$ ) of finite groups are effectively equivalent if there exists an isomorphism $\varphi: G_{1} \rightarrow G_{2}$ such that $\rho_{1}$ and $\rho_{2} \circ \varphi$ are stably equivalent $G_{1}$-representations, write $\rho_{1} \approx_{\text {eff }} \rho_{2}$. Moreover, we say $G_{1} \leq \operatorname{Sym}\left(n_{1}\right)$ and $G_{2} \leq$ $\operatorname{Sym}\left(n_{2}\right)$ are effectively equivalent permutation groups if $G_{1} \hookrightarrow \operatorname{Sym}\left(n_{1}\right)$ and $G_{2} \hookrightarrow$ $\operatorname{Sym}\left(n_{2}\right)$ are effectively equivalent permutation representations.

The permutation groups given in the introduction are effective equivalent. One may think that two transitive permutation groups $G_{1}$ and $G_{2}$ which are effective equivalent already have to be equal. This is not the case as demonstrated in the next example (see also Example 3.1).

Example Let $G=\operatorname{PSL}_{2}(13)$ and consider the actions $\pi_{1}$ and $\pi_{2}$ on the coset spaces $G / H_{1}$ and $G / H_{2}$ where $H_{1}$ is subgroup of $G$ isomorphic to $D_{14}$ and $H_{2}$ a subgroup isomorphic to $D_{12}$. According to Atlas-notation, the permutation characters are $1 a$ $+12 a b c+13 a+14 a a$ and $1 a+12 a b c+13 a a+14 a a$, respectively, see [7]. Thus, $\pi_{1}$ and $\pi_{2}$ are two transitive effective equivalent presentations which are different.

An immediate consequence of the definition of effective equivalence is the following.

Lemma 2.3 If $G_{1}=\left(G, \pi_{1}\right)$ and $G_{2}=\left(G, \pi_{2}\right)$ are two permutation groups such that $P\left(\pi_{1}\right)=P\left(\pi_{2}\right)$, then $G_{1}$ and $G_{2}$ are effective equivalent.

Proof In this case, $\pi_{1}^{-1} \pi_{2}$ is automorphism of $G$, and therefore, $G_{1}$ and $G_{2}$ are effective equivalent.

In $[2,2.3]$ it has been shown that if $\rho$ and $\bar{\rho}$ are two stably equivalent real representations of a finite group $G$, then $P(\rho)$ and $P(\bar{\rho})$ are affinely equivalent. If $\pi_{1}$ and $\pi_{2}$ are effectively equivalent permutation representations, then $\pi_{1}$ and $\pi_{2} \circ \varphi$ are stably equivalent for some isomorphism $\varphi: G_{1} \rightarrow G_{2}$. As $P\left(\pi_{2}\right)=P\left(\pi_{2} \circ \varphi\right)$ the following holds as well:

Theorem 2.4 The permutation polytopes related to two effectively equivalent permutation representations are affinely equivalent.

Notice that Example 2.7 in [2] shows that effectively equivalent permutation representations do not necessarily have lattice equivalent permutation polytopes. This example shows as well that the volumes of two permutation polytopes associated to effectively equivalent permutation representations may be different.

\section{The examples}

In this section, we present an example of a group with two non effectively equivalent permutation representations such that the related permutation polytopes are affinely equivalent. But first, we show the following "almost example". It consists of two permutation groups which are not stably equivalent, but whose permutation polytopes are even equal. It is not really an example to our question as the permutation groups are effectively equivalent. 


\subsection{An "almost example"}

Let $G=\operatorname{Alt}(6)$. Then $G$ contains two different subgroups $H_{1}$ and $H_{2}$ which are both isomorphic to Alt(5), but not conjugate in $G$. We may choose $H_{1}$ as the stabilizer of 1 in the action of $G$ on the set [6] $:=\{1, \ldots, 6\}$. Then $H_{2}$ is transitive on [6]. The group $G$ acts on both coset spaces $G / H_{1}$ and $G / H_{2}$, which yields two permutation representations $\pi_{1}$ and $\pi_{2}$. These representations are not stably equivalent, as they contain different irreducible constituents, see for instance [7], p. 4. On the other hand, as $\left|G: H_{i}\right|=6$ for $i=1,2$, both representations $\pi_{1}$ and $\pi_{2}$ induce embeddings of $G$ into Sym(6). Since in Sym(6) there is only one subgroup isomorphic to Alt(6), it follows that $\pi_{1}(G)=\pi_{2}(G)$. Thus by Lemma 2.3, the two representations $\pi_{1}$ and $\pi_{2}$ are effectively equivalent.

Example 3.1 provides two transitive effective equivalent permutation representations $\pi_{1}$ and $\pi_{2}$ of the same degree which are different. But $\pi_{1}=\pi_{2} \circ \varphi$ for some $\varphi \in \operatorname{Aut}(G)$. We would like to know whether this is always the case:

Question 3.1 Do two transitive effective equivalent permutation representations $\left(G, \pi_{1}\right)$ and $\left(G, \pi_{2}\right)$ of the same degree always only differ by an automorphism of $G ?$

\subsection{The example}

Let $A=\left(\mathbb{Z}_{2}\right)^{2}$ be the direct product of two cyclic groups of order two, $B=\mathbb{Z}_{4}$ and $C=\mathbb{Z}_{3}$ cyclic groups of order 4 and 3 , and let $G:=A \times B \times C$. In the following, we define two different permutation representations of $G$ :

The permutation representation $\pi_{1}$ Let $O_{1}$ be the disjoint union of the right coset spaces $O_{11}:=G / A$ and $O_{12}:=G /(B \times C)$ and let $G$ act by left multiplication on $O_{1}$. Then $O_{11}$ and $O_{12}$ are the $G$-orbits. The kernels of the action of $G$ on $O_{11}$ and $O_{12}$ are $A$ and $B \times C$, respectively. By Lemma 2.7 and Theorem 3.5 of [2], $P\left(\pi_{1}\right)$ is the combinatorial product of an 11-simplex with a 3-simplex.

Notice, if $G=H \times K$ for some subgroups $H$ and $K$ of $G$, then we can extend every irreducible complex representation $\varphi_{H}$ of $H$ to an irreducible complex representation $\varphi$ of $G$ by sending every element of $K$ to the identity. Therefore, we can embed $\operatorname{Irr}_{\mathbb{C}}(H)$ into $\operatorname{Irr}_{\mathbb{C}}(G)$. In this sense, $\operatorname{Irr}_{\mathbb{C}}\left(\pi_{1}\right)$ is the union of $\operatorname{Irr}_{\mathbb{C}}(B \times C)$ and $\operatorname{Irr}_{\mathbb{C}}(A)$. As for an abelian group $\operatorname{Irr}_{\mathbb{C}}(G) \cong G$, see Paragraph 6, 6.4 in [10], it follows that $\operatorname{Irr}_{\mathbb{C}}\left(\pi_{1}\right)$ is the union of two subgroups isomorphic to $\mathbb{Z}_{4} \times \mathbb{Z}_{3}$ and $\left(\mathbb{Z}_{2}\right)^{2}$, respectively.

The permutation representation $\pi_{2}$ Let $\mathrm{O}_{2}$ be the disjoint union of the right coset spaces $O_{21}:=G / B$ and $O_{22}:=G /(A \times C)$ and let $G$ act by left multiplication on $O_{2}$. Then $O_{21}$ and $O_{22}$ are the $G$-orbits. The kernels of the action of $G$ on $O_{21}$ and $O_{22}$ are $B$ and $A \times C$, respectively. By Lemma 2.7 and Theorem 3.5 of [2], $P\left(\pi_{2}\right)$ is again the combinatorial product of an 11-simplex with a 3 -simplex.

Here $\operatorname{Irr}_{\mathbb{C}}\left(\pi_{2}\right)$ is the union of $\operatorname{Irr}_{\mathbb{C}}(A \times C)$ and $\operatorname{Irr}_{\mathbb{C}}(B)$, and therefore, the union of two subgroups isomorphic to $\left(\mathbb{Z}_{2}\right)^{2} \times \mathbb{Z}_{3}$ and $\mathbb{Z}_{4}$.

It follows that $P\left(\pi_{1}\right)$ and $P\left(\pi_{2}\right)$ are affinely equivalent. $\operatorname{In} \operatorname{Irr}_{\mathbb{C}}\left(\pi_{1}\right)$, there is an irreducible representation of order 12 , while every element in $\operatorname{Irr}_{\mathbb{C}}\left(\pi_{2}\right)$ has order at 
most 6 . This shows that the induced real representations $\pi_{1}$ and $\pi_{2} \circ \varphi$ do not contain the same irreducible factors for every automorphism $\varphi \in \operatorname{Aut}(G)$. Thus, $\pi_{1}$ and $\pi_{2}$ are not effectively equivalent.

Remark 3.2 If $G=A \times B \times C$ is an abelian group such that $A$ and $B$ are nonisomorphic groups of the same size, then we can always construct such an example with $\pi_{1}$ and $\pi_{2}$ the actions of $G$ on the unions of coset spaces $O_{1}:=G / A \cup G /(B \times C)$ and $O_{2}:=G / B \cup G /(A \times C)$, respectively.

\section{Characterization of effective equivalence}

Let $G=(G, \pi)$ be a permutation group of degree $n$ with permutation module $V$. Then the affine hull of the polytope $P=P(\pi)$ is

$$
\begin{array}{r}
\operatorname{aff}(\pi(G))=\left\{\sum_{g \in G} \lambda_{g} M_{g} \mid \lambda_{g} \in \mathbb{R}, \sum_{g \in G} \lambda_{g}=1\right\}=E_{n}+U_{\pi}, \text { where } \\
U_{\pi}:=\left\{\sum_{g \in G} \lambda_{g} M_{g} \mid \lambda_{g} \in \mathbb{R}, \sum_{g \in G} \lambda_{g}=0\right\}=\left\{\sum_{g \in G} \lambda_{g}\left(M_{g}-M_{e}\right) \mid \lambda_{g} \in \mathbb{R}\right\} \\
=\left\langle M_{g}-M_{h} \mid g, h \in G\right\rangle_{\mathbb{R}} \leq \operatorname{End}(V) .
\end{array}
$$

The $\mathbb{R}$-vector space $U_{\pi}$ is a $G$-module through the definition

$$
h M:=M_{h} M=\pi(h) M, \quad \text { for } h \in G \text { and } M \in U_{\pi},
$$

as $\pi$ is a group homomorphism from $G$ into $\operatorname{Sym}(n) \leq \mathrm{GL}_{n}(\mathbb{R})$. Notice, that if in particular $M=\pi(g)$, then $h M=\pi(h g)$.

In order to describe the structure of $U_{\pi}$ nicely, we introduce more notation. For $\chi \in \operatorname{Irr}_{\mathbb{R}}(G)$, let $V(\chi)$ be an irreducible $\mathbb{R} G$-module with character $\chi$. Then $\operatorname{End}_{\mathbb{R} G}(V(\chi))$ is either isomorphic to $\mathbb{R}, \mathbb{C}$ or to the quaternions $\mathbb{H}$, and thus $d_{\chi}:=1 / \operatorname{dim}_{\mathbb{R}} \operatorname{End}_{\mathbb{R} G}(V(\chi))$ is either $1, \frac{1}{2}$ or $\frac{1}{4}$. Set

$$
V_{\pi}:=\left\{\sum_{g \in G} \lambda_{g} M_{g} \mid \lambda_{g} \in \mathbb{R}\right\} \quad \text { and } \epsilon_{\pi}:=\sum_{g \in G} M_{g} .
$$

Then $V_{\pi}=U_{\pi} \oplus \mathbb{R} \epsilon_{G}$.

Theorem 4.1 Let $G=(G, \pi)$ be a permutation group of degree $n$ with permutation module $V$. Then $U_{\pi}$ is isomorphic to

$$
\sum_{\chi \in \operatorname{Irr}_{\mathbb{R}}(\pi) \backslash\left\{1_{G}\right\}}\left(d_{\chi} \cdot \chi(1)\right) V(\chi)
$$


as an $\mathbb{R} G$-module, where for $d$ a natural number $d V(\chi)$ is the direct sum of $d$ to $V(\chi)$ isomorphic $\mathbb{R} G$-modules.

Proof Extend $\pi$ linearly to an $\mathbb{R}$-algebra epimorphism $\pi: \mathbb{R} G \rightarrow V_{\pi}$. This then is $\mathbb{R} G$-linear. By Maschke's Theorem, the group algebra $\mathbb{R} G$ is semi-simple. Thus, $V_{\pi}$ is semi-simple as well, and by a theorem by Wedderburn (see for instance [10] Chapter $\mathrm{V}$, Hauptsatz 4.4), we get $\mathbb{R} G=\bigoplus_{i=1}^{m} A_{i}$ with $A_{i}$ simple. Since $A_{i}$ is simple, either $\pi \mid A_{i}$ is injective or $\pi\left(A_{i}\right)=0$. Thus, $V_{\pi}$ is semi-simple as well and we can assume that there is an integer $k \leq m$ such that $V_{\pi} \cong \oplus_{i=1}^{k} A_{i}$. Moreover, we can assume that $A_{1}=\mathbb{R} \epsilon$ with $\epsilon=\sum_{g \in G} g$. Since $\pi(\epsilon)=\epsilon_{\pi}$, we have $U_{\pi}=\oplus_{i=2}^{k} A_{i}$.

Let $\chi \in \operatorname{Irr}_{\mathbb{R}}(G)$ be an irreducible representation of $G$. Then $V(\chi)$ is a composition factor of the $G$-module $V$ if and only if there is an index $1 \leq i \leq k$ such that the $\mathbb{R}$-linear extension of $\chi$ does not vanish on $A_{i}$ or equivalently, $A_{i}=e_{\chi} \mathbb{R} G$ with

$$
e_{\chi}=\sum_{\psi \in \operatorname{Irr}_{\mathbb{C}}(G \mid \chi)} e_{\psi}=\sum_{\psi \in \operatorname{Irr}_{\mathbb{C}}(G \mid \chi)} \frac{1}{|G|} \psi(1) \sum_{g \in G} \overline{\psi(g)} g
$$

the central idempotent corresponding to $\chi$. In this case,

$$
\begin{aligned}
\operatorname{dim}_{\mathbb{R}} A_{i} & \left.=\operatorname{dim}_{\mathbb{R}} \operatorname{End}_{\mathbb{R} G}(V(\chi)) \cdot\left(\operatorname{dim}_{\operatorname{End} \mathbb{R} G}(V(\chi)) V(\chi)\right)\right)^{2} \\
& =\left(1 / d_{\chi}\right) \cdot\left(d_{\chi} \cdot \chi(1)\right)^{2}=d_{\chi} \cdot \chi(1)^{2}
\end{aligned}
$$

and $A_{i} \cong \operatorname{End}_{\mathbb{R}}(V(\chi)) \cong\left(d_{\chi} \cdot \chi(1)\right) V(\chi)$ as an $\mathbb{R} G$-module, see [10], Chapter V, Satz 4.5. Since $\epsilon$ is the central idempotent corresponding to the trivial representation, the claim follows.

The following lemma shows that affine maps between permutation polytopes are always induced by linear maps of their linear hulls.

Lemma 4.2 If $V$ is an $\mathbb{R}$-vector space and $\psi: \operatorname{aff}(P(\pi)) \rightarrow V$, an affine map, then $\psi$ can be uniquely lifted to a linear map $\Psi: V_{\pi} \rightarrow V$.

Proof Since $P(\pi)$ contains a basis of $V_{\pi}$, the space $V_{\pi}$ is the affine hull of $\{0\}$ and $P(\pi)$. Moreover, every element in $\operatorname{aff}(P(\pi))$ is a matrix whose rows and columns all have sum 1 . This shows that $0 \notin \operatorname{aff}(P(\pi))$. Thus, there is a unique affine map $\Psi: V_{\pi} \rightarrow V$ such that $\Psi \mid \operatorname{aff}(P(\pi))=\psi$ and $\Psi(0)=0$. Since $\Psi(0)=0$, the map $\Psi$ is linear.

For $\varphi \in \operatorname{Aut}(G)$, we say that two $G$-modules $U_{1}$ and $U_{2}$ are $\varphi$-isomorphic if there exists an isomorphism $\phi: U_{1} \rightarrow U_{2}$ with $\phi(g u)=\varphi(g) \phi(u)$ for all $u \in U_{1}$ and all $g \in G$. Theorem 4.1 implies the following characterization of the effective equivalent permutation groups:

Theorem 4.3 Let $G_{1}=\left(G, \pi_{1}\right)$ and $G_{2}=\left(G, \pi_{2}\right)$ be two permutation groups. Then the following are equivalent: 
(a) $G_{1}$ and $G_{2}$ are effectively equivalent.

(b) There is a $\varphi \in \operatorname{Aut}(G)$ such that $U_{\pi_{1}}$ and $U_{\pi_{2}}$ are $\varphi$-isomorphic.

(c) There is a $\varphi \in \operatorname{Aut}(G)$ such that $V_{\pi_{1}}$ and $V_{\pi_{2}}$ are $\varphi$-isomorphic.

(d) There is an affine isomorphism $\phi: \operatorname{aff}\left(P\left(\pi_{1}\right)\right) \rightarrow \operatorname{aff}\left(P\left(\pi_{2}\right)\right)$ which maps $P\left(\pi_{1}\right)$ onto $P\left(\pi_{2}\right)$ and which restricted to $\pi_{1}(G)$ is a group homomorphism.

Proof Suppose that (b) holds. Then $U_{\pi_{1}}$ and $U_{\pi_{2} \circ \varphi}$ are isomorphic $G$-modules. By Theorem 4.1, $U_{\pi_{2} \circ \varphi}$ and $U_{\pi_{1}}$ have the same irreducible constituents. Thus, $\pi_{2} \circ \varphi$ and $\pi_{1}$ are stably equivalent and $G_{1}$ and $G_{2}$ are effectively equivalent; so (a) holds. Statements (b) and (c) are equivalent since $V_{\pi_{i}}$ and $U_{\pi_{i}}$ only differ by the trivial $\mathbb{R} G$-module.

Suppose that (a) holds. Then there is an automorphism $\varphi$ of $G$ such that $\pi_{1}$ and $\pi_{2} \circ \varphi$ are stably equivalent. Thus, if $\left(\lambda_{g}\right)_{g \in G}$ is a family of real numbers with $\sum_{g \in G} \lambda_{g}=0$, then by [2] Theorem 2.4

$$
\sum_{g \in G} \lambda_{g} \pi_{1}(g)=0 \quad \text { if and only if } \sum_{g \in G} \lambda_{g} \pi_{2}(\varphi(g))=0 \text {. }
$$

Thus, the map

$$
\phi: \operatorname{aff}\left(P\left(\pi_{1}\right)\right) \rightarrow \operatorname{aff}\left(P\left(\pi_{2}\right)\right), \quad \sum_{g \in G} \lambda_{g} \pi_{1}(g) \mapsto \sum_{g \in G} \lambda_{g} \pi_{2}(\varphi(g)),
$$

where $\sum_{g \in G} \lambda_{g}=1$, is a well-defined affine map.

By the same argument, $\phi$ is injective, and as the image of $\phi$ affinely spans $\operatorname{aff}\left(P\left(\pi_{2}\right)\right)$, the map $\phi$ is surjective as well. As $\pi_{1}, \pi_{2}$, and $\varphi$ are group homomorphisms, the restriction of $\phi$ to $\pi_{1}(G)$ is a group homomorphism onto $\pi_{2}(G)$. This shows that (a) implies (d).

Suppose that (d) holds. We want to show (c). First, note that $\phi$ maps $\pi_{1}(G)$ bijectively onto $\pi_{2}(G)$, since these are vertices of the corresponding polytopes. Thus, $\phi$ induces a group isomorphism between $\pi_{1}(G)$ and $\pi_{2}(G)$ which we will also call $\phi$. Then $\varphi:=\pi_{2}^{-1} \circ \phi \circ \pi_{1}$ is a group automorphism of $G$. If $u=\sum_{g \in G} \lambda_{g} \pi_{1}(g)$ with $\sum_{g \in G} \lambda_{g}=1$ and $x \in G$, then

$$
\begin{aligned}
\phi(x u) & =\phi\left(x \sum_{g \in G} \lambda_{g} \pi_{1}(g)\right)=\phi\left(\sum_{g \in G} \lambda_{g} x \pi_{1}(g)\right)=\sum_{g \in G} \lambda_{g} \phi\left(\pi_{1}(x g)\right) \\
& =\sum_{g \in G} \lambda_{g} \phi\left(\pi_{1}(x)\right) \phi\left(\pi_{1}(g)\right)=\sum_{g \in G} \lambda_{g} \pi_{2}(\varphi(x)) \phi\left(\pi_{1}(g)\right) \\
& =\sum_{g \in G} \lambda_{g} \varphi(x) \phi\left(\pi_{1}(g)\right)=\varphi(x) \sum_{g \in G} \lambda_{g} \phi\left(\pi_{1}(g)\right)=\varphi(x) \phi(u),
\end{aligned}
$$

by the definition of the action of $G$ on $U_{\pi_{1}}$ and $U_{\pi_{2}}$. By Lemma 4.2, we can extend $\phi$ to a linear isomorphism $\Psi: V_{\pi_{1}} \rightarrow V_{\pi_{2}}$, for which one easily sees that $\Psi(g u)=$ $\varphi(g) \Psi(u)$ holds for all $g \in G$ and all $u \in V_{\pi_{1}}$. 
The equivalence between (a) and (d) yields another possibility to describe effective equivalence.

Definition 4.4 If $P$ and $Q$ are two polytopes, $G$ a group which acts as automorphism group on both $P$ and $Q$, then an affine isomorphism $\phi: \operatorname{aff}(P) \rightarrow \operatorname{aff}(Q)$ with $\phi(P)=Q$ is called an affine $G$-isomorphism if there is an automorphism $\varphi$ of $G$ such that $\phi(g x)=\varphi(g) \phi(x)$ holds for all $g \in G$ and all vertices $x$ of $P$.

If $\pi$ is a permutation representation of a finite group $G$, then left multiplication defines a natural action of $G$ on $P(\pi)$ as we saw above. Then the equivalence between (a) and (d) in Theorem 4.3 gives us:

Corollary 4.5 If $G_{1}=\left(G, \pi_{1}\right)$ and $G_{2}=\left(G, \pi_{2}\right)$ are two permutation groups, then they are effectively equivalent if and only if there is an affine $G$-isomorphism between the corresponding permutation polytopes.

Let $G_{i}=\left(G, \pi_{i}\right)$ be permutation groups for $i=1,2$. Suppose that there is an affine isomorphism $\phi: P\left(\pi_{1}\right) \rightarrow P\left(\pi_{2}\right)$. Then we get an isomorphism $\hat{\phi}: \operatorname{Aut}\left(P\left(\pi_{1}\right)\right) \rightarrow$ $\operatorname{Aut}\left(P\left(\pi_{2}\right)\right)$ by setting $\hat{\phi}(g)(x):=\phi\left(g\left(\phi^{-1}(x)\right)\right.$ for $g \in \operatorname{Aut}\left(P\left(\pi_{1}\right)\right)$ and $x \in P\left(\pi_{2}\right)$. For $g \in G$ let $\psi_{i}(g) \in \operatorname{Aut}\left(P\left(\pi_{i}\right)\right)$ be the automorphism defined by $\psi_{i}(g)(u)$ $=\pi_{i}(g) u$ for $u \in \operatorname{aff}\left(P\left(\pi_{i}\right)\right)$. Hence, $\psi_{i}: G \rightarrow \operatorname{Aut}\left(P\left(\pi_{i}\right)\right)$ is a monomorphism. Note that $\psi_{i}(G)$ acts regularly on the set of vertices of $P\left(\pi_{i}\right)$.

Theorem 4.6 $G_{1}$ and $G_{2}$ are effectively equivalent if and only if there is an affine isomorphism $\phi: \operatorname{aff}\left(P\left(\pi_{1}\right)\right) \rightarrow \operatorname{aff}\left(P\left(\pi_{2}\right)\right)$ mapping $P\left(\pi_{1}\right)$ onto $P\left(\pi_{2}\right)$ such that $\hat{\phi}\left(\psi_{1}(G)\right)$ and $\psi_{2}(G)$ are conjugate in $\operatorname{Aut}\left(P\left(\pi_{1}\right)\right)$.

Proof If $G_{1}$ and $G_{2}$ are effectively equivalent, then by Theorem 4.3, there is an affine isomorphism $\phi: \operatorname{aff}\left(P\left(\pi_{1}\right)\right) \rightarrow \operatorname{aff}\left(P\left(\pi_{2}\right)\right)$ with $\Phi\left(P\left(\pi_{1}\right)\right)=P\left(\pi_{2}\right)$ and an isomorphism $\varphi$ of $G$ with $\phi(x u)=\varphi(x) \phi(u)$ for all $x \in G$ and $u \in P\left(\pi_{1}\right)$. Therefore, it follows immediately that $\psi_{2}(G)=\hat{\phi}\left(\psi_{1}(G)\right)$. Now suppose there is $\phi$ : $\operatorname{aff}\left(P\left(\pi_{1}\right)\right) \rightarrow \operatorname{aff}\left(P\left(\pi_{2}\right)\right)$ such that $\phi\left(P\left(\pi_{1}\right)\right)=P\left(\pi_{2}\right)$ and $\psi_{2}(G)$ and $\hat{\phi}\left(\psi_{1}(G)\right)$ are conjugate. We may assume that $\phi\left(\pi_{1}(1)\right)=\pi_{2}(1)$. Since $\psi_{2}(G)$ acts transitively on the vertices of $P\left(\pi_{2}\right)$, we may assume that there is an $a \in \operatorname{Aut}\left(P\left(\pi_{2}\right)\right)_{\pi_{2}(1)}$ such that $\psi_{2}(G)^{a}=\hat{\phi}\left(\psi_{1}(G)\right)$. After replacing $\phi$ by $a^{-1} \circ \phi$ we may assume $\hat{\phi}\left(\psi_{1}(G)\right)=$ $\psi_{2}(G)$. Thus, there is a bijective map $\varphi: G \rightarrow G$ such that $\psi_{2}(\varphi(g))=\hat{\phi}\left(\psi_{1}(g)\right)$ for all $g \in G$. Since $\hat{\phi}, \psi_{1}$ and $\psi_{2}$ are isomorphisms, it follows that $\varphi$ is an automorphism of $G$. Thus, we have for all $g \in G$ and all $u \in \operatorname{aff}\left(P\left(\pi_{1}\right)\right)$ :

$$
\phi\left(\psi_{1}(g)(u)\right)=\phi\left(\psi_{1}(g)\left(\phi^{-1}(\phi(u))\right)\right)=\hat{\phi}\left(\psi_{1}(g)(\phi(u))=\psi_{2}(\varphi(g))(\phi(u)) .\right.
$$

Since $\phi\left(\pi_{1}(g)\right)=\pi_{2}(g)$, we get

$$
\phi\left(\pi_{1}(g)\right)=\phi\left(\psi_{1}(g)\left(\pi_{1}(1)\right)\right)=\psi_{2}(\varphi(g)) \pi_{2}(1)=\pi_{2}(\varphi(g)) .
$$

Thus, $\phi \mid \pi_{1}(G)$ is a group homomorphism and the claim follows by Theorem 4.3.

As a consequence, this lemma establishes Conjecture 5.4 of [2] for our examples. 
Corollary 4.7 If $P\left(\pi_{1}\right) \approx_{\text {aff }} P\left(\pi_{2}\right)$, but $\pi_{1} \not_{\text {eff }} \pi_{2}$, then $\operatorname{Aut}\left(P\left(\pi_{1}\right)\right) \cong$ Aut $\left(P\left(\pi_{2}\right)\right)$ contains two non-conjugate regular subgroups which are isomorphic to $G$. In particular, $\operatorname{Aut}\left(P\left(\pi_{i}\right)\right)>\psi_{i}(G)$.

Proof The polytopes $P\left(\pi_{1}\right)$ and $P\left(\pi_{2}\right)$ are affinely equivalent, while $\pi_{1}$ and $\pi_{2}$ are not effectively equivalent. Thus by Theorem 4.6, the subgroups, $\hat{\phi}\left(\psi_{1}(G)\right)$ and $\psi_{2}(G)$ of $\operatorname{Aut}\left(P\left(\pi_{1}\right)\right)$, are not conjugate and therefore not equal, which yields the assertion.

We wonder whether these results can be used to prove the following.

Question 4.8 Let $G_{1}=\left(G, \pi_{1}\right)$ and $G_{2}=\left(G, \pi_{2}\right)$ be two transitive permutation groups (of the same degree). Are then $P\left(\pi_{1}\right)$ and $P\left(\pi_{2}\right)$ affinely equivalent if and only if $\pi_{1}$ and $\pi_{2}$ are effectively equivalent?

This question has certainly a positive answer if $G$ is abelian, as in this case, every transitive presentation is already regular. If $G_{1}$ and $G_{2}$ are not transitive then the answer to the question is negative as our examples demonstrate. Permutation polytopes for cyclic groups are of importance in statistics, see for instance [9]. Therefore, it would be interesting to know whether they behave more nicely.

Question 4.9 Let $G_{1}=\left(G, \pi_{1}\right)$ and $G_{2}=\left(G, \pi_{2}\right)$ be two cyclic permutation groups. Are then $P\left(\pi_{1}\right)$ and $P\left(\pi_{2}\right)$ affinely equivalent if and only if $\pi_{1}$ and $\pi_{2}$ are effectively equivalent?

\section{Applications}

\subsection{Application of Lemma 2.3 to Alt(6)}

We obtain as an immediate consequence the following well-known fact.

Lemma 5.1 The group $G=\operatorname{Alt}(6)$ has an outer automorphism which interchanges the two conjugacy classes of Alt(5)-subgroups of $G$.

Proof Let $H_{1}$ and $H_{2}$ be two subgroups of Alt(6) of the two conjugacy classes and $\pi_{1}$ and $\pi_{2}$ be as in Subsection 3.1. Then as both representations yield the same permutation polytope, Lemma 2.3 implies that $\left(G, \pi_{1}\right)$ and $\left(G, \pi_{2}\right)$ are effective equivalent. Thus, $\pi_{1}^{-1} \pi_{2}$ is an automorphism of Alt(6) that maps $H_{1}$ onto $H_{2}$.

\subsection{Application of Theorem 4.3 to the example}

In this section we apply our characterization of the effectively equivalent permutation groups given in Theorem 4.3 to give a new, direct, and more geometric proof of the fact that the permutation groups $\left(G, \pi_{1}\right)$ and $\left(G, \pi_{2}\right)$ presented in Example 3.2 are not effectively equivalent. We use the notation introduced in Example 3.2.

Suppose that $\left(G, \pi_{1}\right)$ and $\left(G, \pi_{2}\right)$ are effectively equivalent. Then according to Theorem 4.3, there is an affine isomorphism $\phi: \operatorname{aff}\left(P\left(\pi_{1}\right)\right) \rightarrow \operatorname{aff}\left(P\left(\pi_{2}\right)\right)$ which maps $P\left(\pi_{1}\right)$ to $P\left(\pi_{2}\right)$ and which restricted to $\pi_{1}(G)$ is a group homomorphism. 
Let $H$ be a subgroup of $G$ such that $\pi_{1}(H)$ is the set of vertices of a face of the polytope $P\left(\pi_{1}\right)$. Then $\pi_{1}(H)$ is a subgroup of $\pi_{1}(G)$ which implies that $\phi\left(\pi_{1}(H)\right)$ is a subgroup of $\pi_{2}(G)$. As $\phi$ is an affine isomorphism from aff $\left(P\left(\pi_{1}\right)\right)$ to aff $\left(P\left(\pi_{2}\right)\right)$ as well, the set $\phi\left(\pi_{1}(H)\right)$ is the set of vertices of a face of the polytope $P\left(\pi_{2}\right)$.

Now we count all the faces of $P\left(\pi_{i}\right)$ which have 24 vertices and whose set of vertices is a subgroup of $\pi_{i}(G)$ (for $i=1,2$ ). The polytope $P\left(\pi_{i}\right)$ is the product of an 11-simplex with a 3-simplex. Therefore, every face of $P\left(\pi_{i}\right)$ has the shape $E \times F$ where $E$ is a face of the 11-simplex, and $F$ a face of the 3 -simplex (for $i=1,2$ ).

The faces of $P\left(\pi_{1}\right)$ given by a subgroup of size 24 In this case $G=H_{1} \times H_{2}$, where $H_{1} \cong \mathbb{Z}_{12}$ and $H_{2} \cong \mathbb{Z}_{2}^{2}$; and $P\left(\pi_{1}\right)=P\left(H_{1}\right) \times P\left(H_{2}\right)$. Further $P\left(H_{1}\right)$ is an 11-simplex and $P\left(H_{2}\right)$ a 3-simplex. If $H$ is a subgroup of $G$ such that $\pi_{1}(H)$ is the set of vertices of a face with 24 vertices, then $H=K_{1} \times K_{2}$ such that $K_{i}$ is a subgroup of $H_{i}$ (for $i=1,2$ ) and such that $|H|=\left|K_{1}\right| \cdot\left|K_{2}\right|=24$. Then either $K_{1}=H_{1}$ and $K_{2}$ has order 2 or $K_{1}$ is of order 6 and $K_{2}=H_{2}$. As there is just one subgroup of order 6 in $H_{1}$ and three subgroups of order 2 in $H_{2}$, it follows that the 24-vertex faces which are coming from a subgroup are precisely three faces of the shape of a prism over an 11-simplex and one face which is the product of a 5-simplex with a 3-simplex.

The faces of $P\left(\pi_{2}\right)$ given by a subgroup of size 24 Here we have the factorization $G=M_{1} \times M_{2}$ where $M_{1} \cong \mathbb{Z}_{2}^{2} \times 3$ and $M_{2} \cong \mathbb{Z}_{4}$. The polytope $P\left(\pi_{2}\right)=P\left(M_{1}\right) \times P\left(M_{2}\right)$ is the product of an 11-simplex and a 3-simplex. If $H$ is a subgroup of $G$ such that $\pi_{2}(H)$ is the set of vertices of a face with 24 vertices, then as above $H=K_{1} \times K_{2}$ such that $K_{i}$ is a subgroup of $M_{i}$ (for $i=1,2$ ) and such that $|H|=\left|K_{1}\right| \cdot\left|K_{2}\right|=24$. In this case there are three subgroups of $M_{1}$ of size 6 and precisely one subgroup of $K_{2}$ of size 2 . Therefore the 24-vertex faces which are coming from a subgroup are precisely one prism over an 11-simplex and three faces which are the product of a 5-simplex and a 3-simplex.

This contradicts the fact that $\phi$ maps every face of $P\left(\pi_{1}\right)$ which is induced by a subgroup of $G$ isomorphically onto a face of $P\left(\pi_{2}\right)$ which is induced by a subgroup of $G$. Thus, $\left(G, \pi_{1}\right)$ and $\left(G, \pi_{2}\right)$ are not effectively equivalent.

Another application of Theorem 4.6 and Corollary 2.8 of [2] is that any two isomorphic regular subgroups of $\operatorname{Sym}(n)$ are conjugate in $\operatorname{Sym}(n)$.

Acknowledgments The authors like to thank for support by the DFG through the SFB 701 "Spectral Structures and Topological Methods in Mathematics". Moreover, they thank Benjamin Nill for his very useful comments which helped to improve the paper.

\section{References}

1. Barvinok, A., Stephen, T.: The distribution of values in the quadratic assignment problem. Math. Oper. Res. 28(1), 64-91 (2003)

2. Baumeister, B., Haase, C., Nill, B., Paffenholz, A.: On permutation polytopes. Adv. Math. 222(2), 431-452 (2009)

3. Baumeister, B., Haase, C., Nill, B., Paffenholz, A.: Permutation polytopes of cyclic groups. (preprint) arXiv: 1109.0191. (2011)

4. Baumeister, B., Haase, C., Nill, B., Paffenholz, A.: Polytopes associated to dihedral groups. preprint, arXiv: 1212:4442. (2012) 
5. Billera, L.J., Sarangarajan, A.: The combinatorics of permutation polytopes. In: Billera, L.J. et al. (ed.) Formal power series and algebraic combinatorics. Séries formelles et combinatoire algébrique 1994. Invited lectures presented at the 6th international DIMACS workshop, May 23-27, 1994. Providence, RI: American Mathematical Society. DIMACS, Ser. Discrete Math. Theor. Comput. Sci. 24, 1-23 (1996).

6. Burggraf, K., De Loera, J., Omar, M.: On volumes of permutation polytopes, 2011. Fields Institute Communications 69, (2013)

7. Conway, J.H., Curtis, R.T., Norton, S.P., Parker, R.A., Wilson, R.A.: An ATLAS of Finite Groups. Oxford University Press, Oxford (1985)

8. De Loera, J.A., Liu, F., Yoshida, R.: A generating function for all semi-magic squares and the volume of the Birkhoff polytope. J. Algebr. Comb. 30(1), 113-139 (2009)

9. Eriksson, N., Fienberg, S.E., Rinaldo, A., Sullivant, S.: Polyhedral conditions for the nonexistence of the MLE for hierarchical log-linear models. J. Symb. Comput. 41(2), 222-233 (2006)

10. Huppert, B.: Endliche Gruppen I. Springer, Berlin (1967)

11. Ziegler, G.M.: Lectures on Polytopes. Graduate Texts in Mathematics. Springer, Berlin (1995)

12. Ziegler G.M.: Lectures on 0/1-polytopes. In: Kalai, G. (ed.) et al. Polytopes-combinatorics and computation, DMV-seminar Oberwolfach, Germany, Nov 1997, Basel: Birkhäuser. DMV Semin. 29, 1-41, 2000 